\title{
Denkbar schlechtes Zeugnis für Transfette
}

\author{
Im Juni hat die US-Regulierungsbehörde FDA einen Aktionsplan vorgestellt, um un- \\ gesättigte Transfettsäuren weitestgehend aus Lebensmitteln zu verbannen. Eine Meta- \\ analyse zeigt, dass „Transfette" tatsächlich für höhere Mortalität verantwortlich sind.
}

_ In vielen Lebensmitteln sind gesättigte Fettsäuren sowie Transfettsäuren enthalten, u. a. in Milchprodukten und im Fleisch von Wiederkäuern. Sie entstehen auch beim Erhitzen von Pflanzenölen mit einem hohen Gehalt an mehrfach ungesättigten Fettsäuren. In einer Metaanalyse wurde der Zusammenhang zwischen den beiden Fettgruppen mit der Gesamtmortalität, kardiovaskulären Erkrankungen, speziell koronaren Herzerkrankungen, und deren Mortalität, Schlaganfall und Diabetes mellitus Typ 2 genauer unter die Lupe genommen. $\mathrm{Zu}$ gesättigten Fettsäuren konnten bis zu zwölf, zu Transfettsäuren bis zu sechs prospektive Kohortenstudien eingeschlossen werden.

Für die gesättigten Fettsäuren konnten keine signifikanten Zusammenhänge mit der Gesamtmortalität, der kardiovaskulären Mortalität, koronaren Herzerkrankungen und deren Mortalität, Schlaganfall oder Typ-2-Diabetes er- mittelt werden. Dagegen geht der Verzehr von Transfetten mit einer Erhöhung der Gesamtmortalität um 34\%, dem Auftreten koronarer Herzerkrankungen um $21 \%$ und der dadurch verursachten Mortalität um 28\% einher. Das Risiko für Schlaganfall und Diabetes ist nicht signifikant erhöht. Industriell hergestellte Transfette sind zudem mit einer höheren Mortalität aufgrund koronarer Herzerkrankungen vergesellschaftet.

Unter Berücksichtigung methodischer Schwächen schlussfolgern die Autoren, dass die Ergebnisse von schwacher Evidenz sind und entsprechend vorsichtig interpretiert werden müssen.

- de Souza RJ, Mente A, Maroleanu A et al. Intake of saturated and trans unsaturated fatty acids and risk of all cause mortality, cardiovascular disease, and type 2 diabetes: systematic review and meta-analysis of observational studies. BMJ. 2015;351:h3978

\section{KOMMENTAR}

Die Arbeit reiht sich in die Flut von Metaanalysen zum gleichen Thema ein und bestätigt frühere Ergebnisse. Die Autoren interpretieren sehr vorsichtig und legen methodische Schwächen sehr gut offen. Letztere führen leider dazu, dass die Daten nicht allzu verlässlich sind und kaum Schlussfolgerungen gezogen werden können.

Zurecht fragen die Autoren auch, womit eine Reduktion von gesättigten Fettsäuren und Transfettsäuren kompensiert werden soll. Dazu haben Studien gezeigt, dass der Austausch von gesättigten durch mehrfach ungesättigte Fettsäuren das Risiko für koronare Herzerkrankungen senkt. Dies funktioniert nicht, wenn einfach ungesättigte Fette oder Kohlenhydrate eingesetzt werden.

Die Deutsche Gesellschaft für Ernährung empfieh/t, weniger als 10\% des Energiebedarfs durch gesättigte Fette und weniger als 1\% durch Transfette zu decken. Beim Verzehr von Pommes, Chips oder Süßwaren ist somit Vorsicht geboten - was natürlich im Hinblick auf das Gewichtsmanagement sowieso zu empfehlen ist.

Dr. rer. nat. C. Holzapfel

\section{Speichelstein verursacht dumpfen Schmerz im Bereich der rechten Zungenhälfte}

Eine 77-jährige Frau hatte seit etwa sechs Monaten einen dumpfen Schmerz im Bereich der rechten Zungenhälfte. Die Inspektion der Zunge war unauffällig, allerdings erkannte man einen Speichelstein, der aus dem Ausführungsgang der Glandula sublingualis rechts ragte. Die Umgebung der Caruncula sublingualis rechts war gerötet und geschwollen (Abb. A). Der Speichelstein konnte mit einer Pinzette einfach entfernt werden, aus dem Gang entleerte sich Pus und normaler Speichel. Der verkalkte Stein wies eine Länge von 1,6 cm auf (Abb. B). Bei der Kontrolluntersuchung eine Woche später ging es der Patientin gut, der Speichelfluss war normal
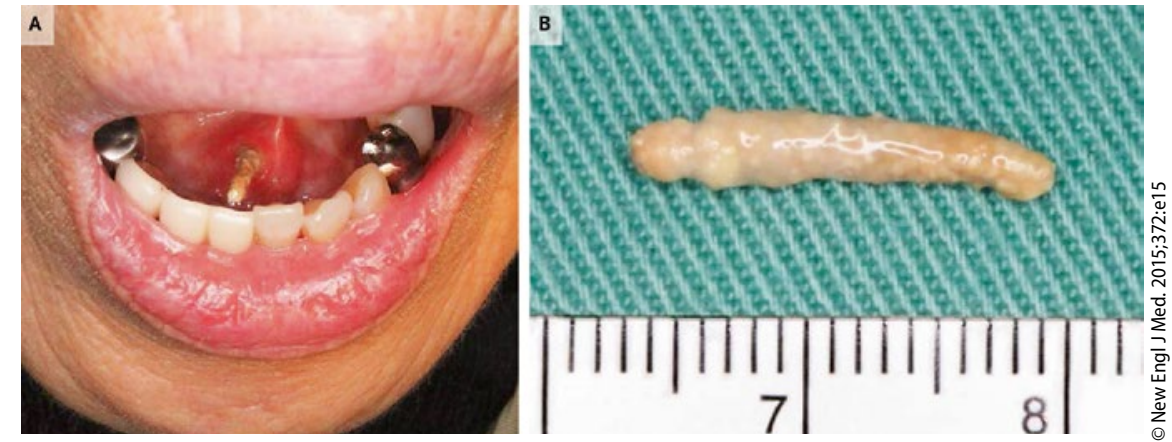

Speichelstein im Ausführungsgang der Glandula sublingualis (A), nach Entfernung (B).

und die Schmerzen im Zungenbereich waren verschwunden.
- Adachi M, MotohashiM (madachi_dds_phd@yahoo.co.jp). Passage of a sialolith. New Engl J Med. 2015;372:e15 